\title{
Specific muscle strength is reduced in facioscapulohumeral dystrophy: An MRI based musculoskeletal analysis
}

\author{
Marco A. Marra ${ }^{\mathrm{a}, 1, *}$, Linda Heskamp ${ }^{\text {b,1 }}$, Karlien Mul c , Saskia Lassche ${ }^{\text {, }}$ \\ Baziel G.M. van Engelen ${ }^{\mathrm{c}}$, Arend Heerschap ${ }^{\mathrm{b}}$, Nico Verdonschot ${ }^{\mathrm{a}, \mathrm{d}}$ \\ ${ }^{a}$ Orthopaedic Research Laboratory, Radboud Institute for Health Sciences, Radboud university medical center, P.O. Box 9101, 6500 HB Nijmegen, \\ The Netherlands \\ ${ }^{\mathrm{b}}$ Department of Radiology and Nuclear Medicine, Radboud university medical center, P.O. Box 9101, 6500 HB Nijmegen, The Netherlands \\ ${ }^{\mathrm{c}}$ Department of Neurology, Radboud university medical center, P.O. Box 9101, 6500 HB Nijmegen, The Netherlands \\ ${ }^{d}$ Department of Biomechanical Engineering, University of Twente, Postbus 217, 7500 AE Enschede, The Netherlands
}

Received 28 July 2017; received in revised form 28 September 2017; accepted 26 November 2017

\begin{abstract}
The aim was to test whether strength per unit of muscle area (specific muscle strength) is affected in facioscapulohumeral dystrophy (FSHD) patients, as compared to healthy controls. Ten patients and ten healthy volunteers underwent an MRI examination and maximum voluntary isometric contraction measurements (MVICs) of the quadriceps muscles. Contractile muscle volume, as obtained from the MR images, was combined with the MVICs to calculate the physiological cross-sectional area (PCSA) and muscle strength using a musculoskeletal model. Subsequently, specific strength was calculated for each subject as muscle strength divided by total PCSA. FSHD patients had a reduced quadriceps muscle strength (median(1st quartile-3rd quartile): 2011 (905.4-2775) N vs. 5510 (4727-8321) N, $p<0.001$ ) and total PCSA (83.6 (62.3$124.8) \mathrm{cm}^{2}$ vs. $\left.140.1(97.1-189.9) \mathrm{cm}^{2}, p=0.015\right)$ compared to healthy controls. Furthermore, the specific strength of the quadriceps was significantly lower in patients compared to healthy controls $\left(20.9(14.7-24.0) \mathrm{N} / \mathrm{cm}^{2} v s .41 .9(38.3-49.0) \mathrm{N} / \mathrm{cm}^{2}, p<0.001\right)$. Thus, even when correcting for atrophy and fatty infiltration, patients with FSHD generated less force per unit area of residual muscle tissue than healthy controls. Possible explanations include impaired force propagation due to fatty infiltration, reduced intrinsic force-generating capacity of the muscle fibers, or mitochondrial abnormalities leading to impaired energy metabolism.
\end{abstract}

(C) 2017 Elsevier B.V. All rights reserved.

Keywords: Facioscapulohumeral muscular dystrophy; Specific strength; Quantitative MRI; Musculoskeletal modeling; Intrinsic weakness

\section{Introduction}

Facioscapulohumeral muscular dystrophy (FSHD) is a hereditary disease characterized by progressive loss of muscle strength, starting in the face, shoulder and upper arm region [1]. In addition, the trunk and lower extremity muscles are frequently involved [1]. Histopathological changes can be very diverse, ranging from mild myopathic features to overt dystrophic changes with fatty infiltration and fibrosis [2]. Magnetic Resonance Imaging (MRI) of the lower extremity

\footnotetext{
* Corresponding author. Radboud University Medical Center, 611 Orthopaedic Research Laboratory, P.O. Box 9101, 6500 HB Nijmegen, The Netherlands.

E-mail address: mamarra@outlook.com (M.A. Marra).

${ }^{1}$ Marco A. Marra and Linda Heskamp contributed equally to this work and should be considered therefore co-first authors.
}

muscles reveals that muscles are affected by fatty infiltration in a specific pattern [3-5]. For example, in the thigh there is early involvement of the hamstrings, adductors and rectus femoris, whereas the vasti muscles usually become involved later in the disease course.

To aid the development of new treatments for FSHD it is important to understand why muscles of FSHD patients are weak. Muscle strength depends on the physiological crosssectional area (PCSA) [6], i.e. the cross-sectional area perpendicular to the direction of the muscle fibers. In FSHD, fatty infiltration, fibrosis and muscle fiber atrophy affect the muscle tissue and are assumed to result in a reduced PCSA and contractile muscle volume, i.e. the portion of the muscle able to generate force. Therefore, fatty infiltration, atrophy and fibrosis may all contribute to muscle weakness. Janssen et al. [3] indeed showed that the amount of contractile muscle tissue in the quadriceps muscles of FSHD patients is related to muscle 
strength. Furthermore, it has been suggested that reduced specific muscle strength, defined as strength per unit area of contractile muscle tissue, is an additional cause of muscle weakness in FSHD [7,8]. However, Bachasson et al. [7] did not include imaging of the quadriceps muscles and some of us [8] investigated specific muscle strength only on a single muscle fiber level.

In this study, we investigate whether the specific strength of the quadriceps muscles is affected in FSHD patients as compared to healthy controls. For this aim we evaluated contractile volume, PCSA and strength of quadriceps muscles by combining quantitative MRI, quantitative muscle assessment and musculoskeletal modeling.

\section{Material and methods}

\subsection{Participants}

FSHD patients and healthy control subjects were retrospectively recruited from two cohorts. The FSHD patients were selected from a larger database of 140 FSHD patients who are participating in a prospective cohort study (FSHD-FOCUS study, Nijmegen, The Netherlands). The control cohort consisted of a group of ten healthy volunteers who participated in the TLEMsafe project dataset (FP7-ICT-2009-4). All subjects performed a maximum voluntary isometric contraction (MVIC) of the knee extensor muscles and underwent an MRI examination of the quadriceps muscles. On the day of MVIC examinations, all patients performed also manual muscle testing, 6-minute walking test, motor function measure, and spirometry, as part of the FSHD-FOCUS study protocol. No other tests were performed by the healthy controls on the examination days. Gender, age, height, and weight have been recorded for both groups. FSHD disease severity were assessed with a 10-point clinical severity score (Ricci score) [9]. For the present study, FSHD patients and health volunteers were included if the MVIC measurement was performed correctly, and the MR images of the lower extremity covered the entire span of the quadriceps muscles and contained no artifacts, like movement or failed reconstruction of the fat and water images obtained via a 2 pt-Dixon sequence.

This study was conducted according to the principles of the Declaration of Helsinki (version October 2013) and in accordance with the Medical Research Involving Human Subjects Act (WMO). In both groups, informed consent was obtained from each participant.

\subsection{Experimental measurements}

\subsubsection{MR imaging}

All subjects were examined on a 3T MR system (TIM Trio, Siemens, Erlangen, Germany) using a ${ }^{1} \mathrm{H}$ spine coil combined with phased array coils placed around the leg.

In the FSHD patients MR data of the thigh were recorded with a 2pt-Dixon sequence to quantify the amount of fatty infiltration (repetition time (TR): $10 \mathrm{~ms}$, echo time (TE) in-phase: $2.45 \mathrm{~ms}$, TE out-phase: $3.675 \mathrm{~ms}$, flip angle (FA): $3^{\circ}$, voxel size: $1.36 \times 1.36 \times 5.00 \mathrm{~mm}^{3}, 1$ or 2 stacks with number of slices: 72). A fat fraction map, ranging from zero to one, was calculated for each slice from the reconstructed water and fat images by dividing the signal intensities of every voxel in the fat image by the signal intensities of the same voxel in the fat and water images summed together, as $\mathrm{F} /(\mathrm{F}+\mathrm{W})$.

The dataset of healthy volunteers contained T1-weighted MR images of the lower extremity from hip to ankle (TR/TE: $545 / 9 \mathrm{~ms}$, voxel size: $1.04 \times 1.04 \times 3.00 \mathrm{~mm}^{3}$, number of slices: 400). These T1-weighted data did not allow for quantitative fat measurements. Visual inspection of fatty infiltration on T1-weighted images did not show any pathological fatty infiltration of the quadriceps muscles in any of the healthy subjects. Therefore, for the healthy group, fat fraction values were assumed equal to $8.4 \%, 7.1 \%, 6.7 \%$ and $7.5 \%$ in the rectus femoris, vastus intermedius, vastus lateralis, and vastus medialis respectively, based on our previous measurements in a separate group of ten healthy volunteers (unpublished data, mean (1st quartile-3rd quartile); age: 41.5 (38-56) years old; BMI: $\left.24.0(21.6-24.9) \mathrm{kg} / \mathrm{m}^{2}\right)$.

\subsubsection{Strength assessment in the FSHD group}

Maximum voluntary isometric contraction of the knee extensor muscles was assessed in the dominant leg of FSHD patients. Each participant was seated with both hips and knees flexed at $90^{\circ}$ on a fixed quadriceps dynamometer. The ankle of the dominant leg was secured to the dynamometer using Velcro straps and located at a distance of $24.5 \mathrm{~cm}$ from the knee joint center. In this position, the participants were instructed to extend their leg, pushing maximally against the dynamometer. Isometric force was recorded and was fed back visually to the subject on a computer monitor. The test consisted of three consecutive knee extensor MVICs of 4 seconds each, with a resting period of 20 seconds between the tests. Hand support was not allowed throughout the test. Strong verbal and visual encouragements were employed to motivate the participants to deliver their maximal muscle effort. The maximum out of three measured forces was chosen as the final MVIC force. The MVIC force was then multiplied by the knee-to-sensor distance, resulting in the MVIC torque $(\tau)$.

\subsubsection{Strength assessment in the control group}

The MVIC in healthy controls was assessed using a Biodex Dynamometer setup (Biodex Medical Systems, Inc., Shirley, NY, USA). In brief, participants were seated on the chair of the device with both hips and knees flexed by $90^{\circ}$. Belts were placed around the thorax and the thigh and the ankle of the measured leg were tightened. Only the dominant leg was measured, similarly to the FSHD group. Three consecutive MVICs of 6 seconds each were performed, with a resting period of 20 seconds between the tests. Visual feedback and verbal motivation were employed. The highest of the three measured torques was taken as the final outcome.

A post hoc experiment was carried out in a separate group of 9 healthy volunteers to identify possible systematic differences in the MVIC measures between FSHD and control group, owing to different measurement devices. Bland-Altman analysis revealed a measurement bias and an intraclass correlation coefficient of $1.0 \mathrm{Nm}$ and 0.94 , respectively. Hence, 
T1 weighted image Healthy Control

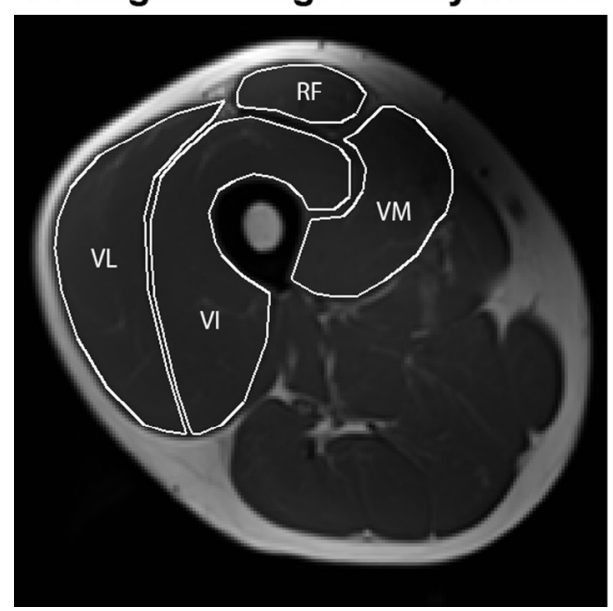

Fat Fraction map FSHD patient

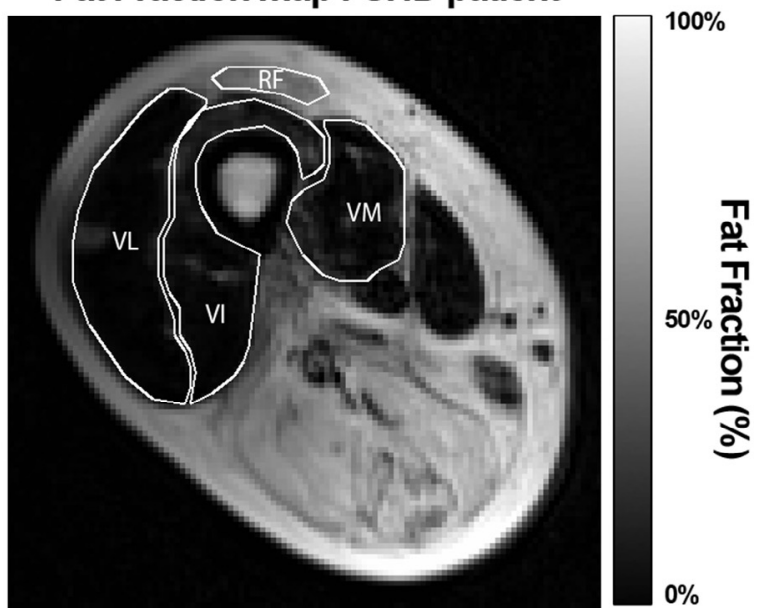

Fig. 1. A) T1-weighted MR image of the thigh muscles of a healthy volunteer. B) Fat fraction map of the thigh muscles of an FSHD patient acquired by a 2 pt Dixon method. Regions of interest are drawn around the m. rectus femoris (RF), m. vastus lateralis (VL), m. vastus intermedius (VI) and $m$. vastus medialis (VM).

we did not find any systematic difference in MVIC assessment when using two different measurement devices.

\subsection{Data analysis}

\subsubsection{MRI and segmentation and volume/fat fraction}

MR images were analyzed using the Medical Image Processing, Analysis, and Visualization software package (MIPAV, Center for Information Technology, National Institutes of Health, obtainable at http://mipav.cit.nih.gov) and MATLAB (version R2014B, The Mathworks, Inc. Natick, Massachusetts, United States). Muscle contours of the four quadriceps muscles were manually delineated every $2.5 \mathrm{~cm}$ in the axial direction from the most distal MRI slice to the most proximal MR slice (see Fig. 1). Caution was taken to avoid the inclusion of subcutaneous fat, fascia, and large blood vessels. Anatomical muscle volume $\left(V_{a}\right)$ was determined as the number of voxels within the muscle mask across all slices multiplied by the voxel area and by the distance between slices of $2.5 \mathrm{~cm}$ [10]. In FSHD patients, an average fat fraction $\left(f_{\text {fat }}\right)$ was calculated for each of the quadriceps muscles over the voxels in the muscle mask across all slices at which the quadriceps muscles were delineated.

It was assumed that the fatty infiltrate cannot generate force. Thus, for each muscle, the contractile muscle volume $\left(V_{c}\right)$ was determined as

$V_{c}=V_{a} \cdot\left(1-f_{\text {fat }}\right)$

where $V_{a}$ is the anatomical muscle volume.

\subsubsection{Calculation of specific muscle strength}

Maximal force-generating capacity in a skeletal muscle, or muscle strength $(S$, in Newton $(\mathrm{N}))$, is proportional to the muscle physiological cross-sectional area (PCSA, in $\mathrm{cm}^{2}$ ). $S$ and PCSA are related by the specific muscle strength $(\sigma$, in $\left.\mathrm{N} / \mathrm{cm}^{2}\right)$ :
$S=\sigma \cdot \mathrm{PCSA}$

$S$ is also related to the experimentally measured torque $(\tau$, in $\mathrm{Nm})$ by the relation:

$\tau=r_{\text {knee }} \cdot S$

To derive $r_{\text {knee }}$ and PCSA of the quadriceps muscles in all participants we used a musculoskeletal model. Briefly, a standard model was obtained from the AnyBody Managed Model Repository (AMMR 1.6.4) in the AnyBody Modeling System (AMS, version 6.0.5, AnyBody Technology A/S, Aalborg, Denmark) [11]. Body height was used to scale the body segments dimensions uniformly. Since the moment arm of a muscle about a joint depends on the angle of that joint, the model was configured in a sitting position similar to the one used in the experiments, with both hips and knees flexed by $90^{\circ}$, see Fig. 2. Existing algorithms available in the AMMR were used to calculate $r_{\text {knee }}$ for each subject, using the tendon excursion method [13].

The PCSA of each of the quadriceps muscles was calculated from the contractile volume $\left(V_{c}\right)$ divided by the optimal fiber length $\left(l^{\circ}\right)$ and corrected for the pennation angle $(\varphi)$ using the relationship [14]

$\operatorname{PCSA}\left(\mathrm{cm}^{2}\right)=\frac{V_{c} \cos \varphi}{l^{0}}$

in which $l^{0}$ and $\varphi$ were derived from a dataset of architectural properties of cadaveric lower extremity muscles [12]. The total PCSA of the quadriceps $\left(\mathrm{PCSA}_{\mathrm{tot}}\right)$ was calculated as the sum of the individual quadriceps muscles PCSAs. Finally, a specific muscle strength for each participant was calculated from Eq. (2), dividing $S$ by PCSA $_{\text {tot }}$.

\subsection{Statistical analysis}

Statistical analysis was performed using Graphpad Prism 5 for Windows (version 5.03, Graphpad Software, San Diego, 


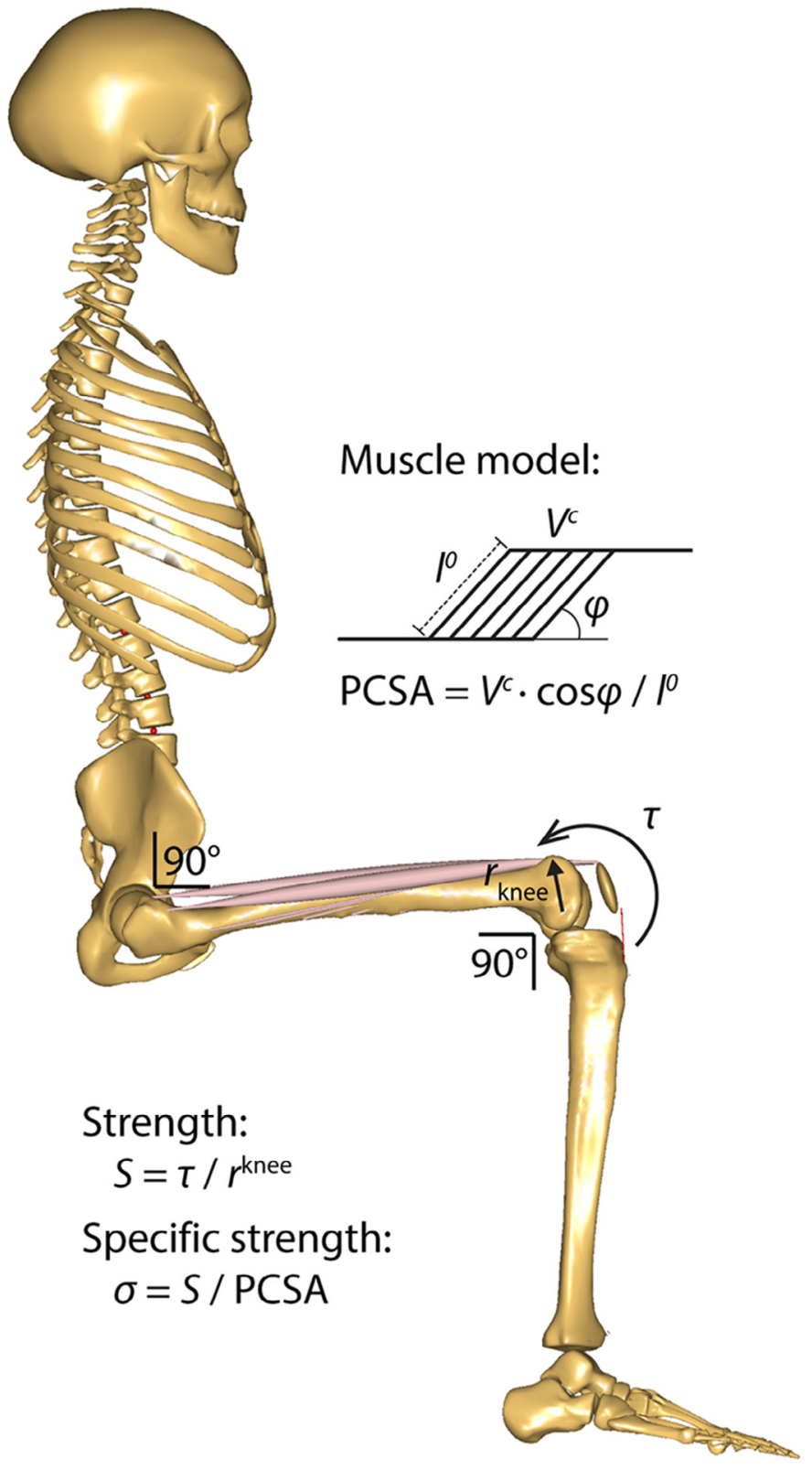

Fig. 2. A view of the musculoskeletal model used to calculate the specific strength $(\sigma)$. The model was adapted from the 'Standing Model' as available in the AnyBody Managed Model Repository (AMMR 1.6.4) in the AnyBody Modeling System (AMS, version 6.0.5, AnyBody Technology A/S, Aalborg, Denmark). The model was scaled based on the height of each subject and measurements of muscle volume. Contractile quadriceps muscle volumes $\left(V_{c}\right)$ were measured by anatomical MRI and corrected using fat fraction maps. Pennation angle $(\varphi)$ and optimal fiber length $\left(l^{0}\right)$ values were extracted from the literature [12]. The average moment arm of quadriceps muscles $\left(r_{\text {knee }}\right)$ was calculated at 90 degrees of knee flexion using the tendon excursion method.

California, USA). Two-tailed unpaired Student's t tests were used to compare the characteristics of FSHD patients and healthy volunteers. Not normally distributed characteristics (according to Shapiro-Wilk test) were compared using the Mann-Whitney U test. Significance level was set to 0.05 . The same test was used to compare $\tau, S, V_{a}, V_{c}, f_{\text {fat }}$ PCSA, and $\sigma$. The relation between $S$ and PCSA $_{\text {tot }}$ in both the FSHD and control group was analyzed using Pearson product moment correlation analysis. We used data on specific muscle strength on 20 adult healthy subjects for use in a sample size calculation for our study because we found no sufficient information regarding specific strength variance over the FSHD population [15]. They reported specific muscle strength to be equal to (mean $\pm \mathrm{SD}$ ) $56 \pm 11 \mathrm{~N} / \mathrm{cm}^{2}$. Setting a power of 0.8 and a Type I error of 0.05 , results in a sample size of 10 patients (and 10 controls) to be able to detect a significant difference of $25 \%$ in specific strength, which we consider to be clinically relevant. Due to the small sample size, data are presented as median (1st quartile-3rd quartile).

\section{Results}

\subsection{Participants characteristics}

Ten FSHD patients and ten healthy controls met our inclusion criteria and were included in the analysis. Both groups were comparable in age, height, weight, and BMI (see Table 1 for their characteristics). Four out of the ten FSHD patients included in this study performed the muscle strength on the same day as the MRI examination. In the other six patients the examinations took place between thirty days and eleven weeks apart. The examinations in healthy controls were completed no more than two months apart.

\subsection{Quantitative MRI and strength measurements}

The MRI data revealed that fat fraction was significantly increased in quadriceps muscles of FSHD patients relative to normal values $(p<0.01)$. The rectus femoris and vastus medialis of FSHD patients had a reduced anatomical volume compared to the control group (rectus femoris: $\mathrm{p}=0.005$; vastus medialis: $p=0.029$ ) (Table 2). Furthermore, the contractile volume of all quadriceps muscles, except the vastus lateralis, was lower in FSHD patients compared to healthy controls (rectus femoris: $\mathrm{p}=0.004$; vastus intermedius: $\mathrm{p}=0.043$; vastus medialis: $\mathrm{p}=0.019$, Table 2). Maximum voluntary isometric contraction torque was significantly lower in FSHD patients compared to healthy controls $(p<0.001$; Table 2).

\subsection{Musculoskeletal modeling: PCSA and specific strength calculation}

Quadriceps muscle strength in FSHD patients was significantly lower than in healthy controls (FSHD: 2011

Table 1

Characteristics of patients with facioscapulohumeral dystrophy (FSHD) and healthy controls.

\begin{tabular}{lll}
\hline & FSHD $(\mathrm{n}=10)$ & Controls $(\mathrm{n}=10)$ \\
\hline Female $(\%)$ & 50.0 & 50.0 \\
Age (years) & $56.0(47.8,66.5)$ & $35.5(24.5,57.5)$ \\
Height $(\mathrm{cm})$ & $174.0(168.8,177.3)$ & $176.0(163.8,183.5)$ \\
Weight $(\mathrm{kg})$ & $72.0(54.8,87.5)$ & $76.8(62.9,84.9)$ \\
BMI $\left(\mathrm{kg} / \mathrm{m}^{2}\right)$ & $23.3(19.9,27.2)$ & $24.5(21.7,26.4)$ \\
Ricci score & $7.5(2.8,8.0)$ & - \\
\hline
\end{tabular}

Data are shown as median (1st quartile, 3rd quartile). 
Table 2

Maximum voluntary isometric contraction (MVIC) torque, calculated strength, anatomical volume, fat fraction, contractile volume, physiological cross-sectional area (PCSA) and specific strength of quadriceps muscles in patients with facioscapulohumeral dystrophy (FSHD) and healthy controls.

\begin{tabular}{|c|c|c|c|c|}
\hline & FSHD $(n=10)$ & Controls $(n=10)$ & $p$-values & \\
\hline \multicolumn{5}{|l|}{$\overline{M V I C}$} \\
\hline Measured torque (Nm) & $63.8(28.5,91.3)$ & $185.4(146.1,286.2)$ & $<0.001$ & $* * *$ \\
\hline Calculated strength $(\mathrm{N})$ & $2011(905.4,2775)$ & $5510(4727,8321)$ & $<0.001$ & $* * *$ \\
\hline \multicolumn{5}{|l|}{ Anatomical volume } \\
\hline Total $\left(\mathrm{cm}^{3}\right)$ & $1046(755.3,1361)$ & $1515(1049,2039)$ & 0.029 & $*$ \\
\hline Rectus femoris $\left(\mathrm{cm}^{3}\right)$ & $107.2(44.1,144.7)$ & $203.5(139.5,322.4)$ & 0.005 & $* *$ \\
\hline Vastus lateralis $\left(\mathrm{cm}^{3}\right)$ & $408.8(346.6,489)$ & $535.4(329.0,697.0)$ & 0.393 & ns \\
\hline Vastus intermedius $\left(\mathrm{cm}^{3}\right)$ & $322.0(218.1,368.3)$ & $346.6(317.9,510.6)$ & 0.123 & ns \\
\hline Vastus medialis $\left(\mathrm{cm}^{3}\right)$ & $212.1(103.2,326.4)$ & $358.7(272.4,497.9)$ & 0.029 & $*$ \\
\hline \multicolumn{5}{|l|}{ Fat fraction } \\
\hline Total (\%) & $12.2(9.3,19.2)$ & $7.3(7.2,7.3)$ & 0.001 & $* *$ \\
\hline Rectus femoris (\%) & $17.3(8.2,45.0)$ & $8.4^{\mathrm{a}}$ & 0.020 & $*$ \\
\hline Vastus lateralis (\%) & $10.5(8.7,15.5)$ & $7.1^{\mathrm{a}}$ & 0.011 & $*$ \\
\hline Vastus intermedius (\%) & $10.4(8.0,20.9)$ & $6.7^{\mathrm{a}}$ & 0.002 & $*$ \\
\hline Vastus medialis $(\%)$ & $15.4(8.8,35.1)$ & $7.5^{\mathrm{a}}$ & 0.011 & $*$ \\
\hline \multicolumn{5}{|l|}{ Contractile volume } \\
\hline Total $\left(\mathrm{cm}^{3}\right)$ & $862.4(639.7,1264)$ & $1405(973.4,1891)$ & 0.019 & $*$ \\
\hline Rectus femoris $\left(\mathrm{cm}^{3}\right)$ & $88.7(24.2,128.9)$ & $186.4(127.8,295.3)$ & 0.004 & $* *$ \\
\hline Vastus lateralis $\left(\mathrm{cm}^{3}\right)$ & $353.1(312.8,456.2)$ & $497.4(305.7,647.5)$ & 0.315 & ns \\
\hline Vastus intermedius $\left(\mathrm{cm}^{3}\right)$ & $284.4(176.5,331.6)$ & $323.4(296.6,476.4)$ & 0.043 & $*$ \\
\hline Vastus medialis $\left(\mathrm{cm}^{3}\right)$ & $165.9(71.3,302.2)$ & $331.8(252.0,460.5)$ & 0.019 & * \\
\hline \multicolumn{5}{|l|}{$P C S A$} \\
\hline Total $\left(\mathrm{cm}^{2}\right)$ & $83.6(62.3,124.8)$ & $140.1(97.1,189.9)$ & 0.015 & $*$ \\
\hline Rectus femoris $\left(\mathrm{cm}^{2}\right)$ & $11.3(3.1,16.5)$ & $23.8(16.3,37.8)$ & 0.004 & $* *$ \\
\hline Vastus lateralis $\left(\mathrm{cm}^{2}\right)$ & $33.7(29.9,43.6)$ & $47.5(29.2,61.8)$ & 0.315 & ns \\
\hline Vastus intermedius $\left(\mathrm{cm}^{2}\right)$ & $28.6(17.7,33.3)$ & $32.5(29.8,47.8)$ & 0.043 & $*$ \\
\hline Vastus medialis $\left(\mathrm{cm}^{2}\right)$ & $14.9(6.4,27.1)$ & $29.8(22.6,41.4)$ & 0.019 & $*$ \\
\hline Calculated specific strength $\left(\mathrm{N} / \mathrm{cm}^{2}\right)$ & $20.9(14.7,24.0)$ & $41.9(38.3,49.0)$ & $<0.0001$ & $* * *$ \\
\hline
\end{tabular}

Data are shown as median (1st quartile, 3rd quartile). MVIC: maximum voluntary isometric contraction; PCSA: physiological cross-sectional area.

a Fat fraction of individual muscles of healthy controls is based on unpublished experimental data.

$* p<0.05, * * p<0.01, * * * p<0.001$.

(905.4-2775) N, healthy controls: $5510(4727-8321) \mathrm{N}$, $p<0.001$; Table 2). Total PCSA, i.e. cross-sectional area perpendicular to the muscle fiber direction, of the quadriceps in FSHD patients was significantly lower than in healthy controls (FSHD: $83.6(62.3-124.8) \mathrm{cm}^{2}$, healthy controls: 140.1 (97.1189.9) $\mathrm{cm}^{2}, p=0.015$; Table 2). Total PCSA correlated very strongly with the quadriceps muscle strength (Fig. 3; FSHD: $\mathrm{r}=0.95, p<0.001$; healthy controls: $\mathrm{r}=0.86, p=0.001$ ). Specific muscle strength, i.e. strength per unit of PCSA, was significantly lower in FSHD patients compared to healthy controls (FSHD: $20.9(14.7-24.0) \mathrm{N} / \mathrm{cm}^{2}$; healthy controls: 41.9 (38.3-49.0) N/ $\mathrm{cm}^{2}, p<0.0001$, Fig. 4). Hence, specific strength was reduced by $56 \%$ in FSHD patients relative to controls.

\section{Discussion}

Our study shows that specific muscle strength is reduced more than $50 \%$ in our group of FSHD patients relative to healthy controls. In addition, contractile muscle volume and PCSA are significantly reduced. Therefore, it can be concluded that muscle weakness in FSHD is not only caused by a reduced amount of available contractile muscle tissue, but also by reduced function of the residual muscle tissue.

Muscle weakness in FSHD is partly caused by the reduction in the contractile muscle volume and PCSA. The smaller contractile muscle volume is firstly caused by the infiltration of fat. Furthermore, atrophy plays a role, because the anatomical muscle volume of the rectus femoris and vastus medialis is also reduced. The most important finding of our study is that the

\section{Strength vs. PCSA ${ }_{\text {tot }}$}

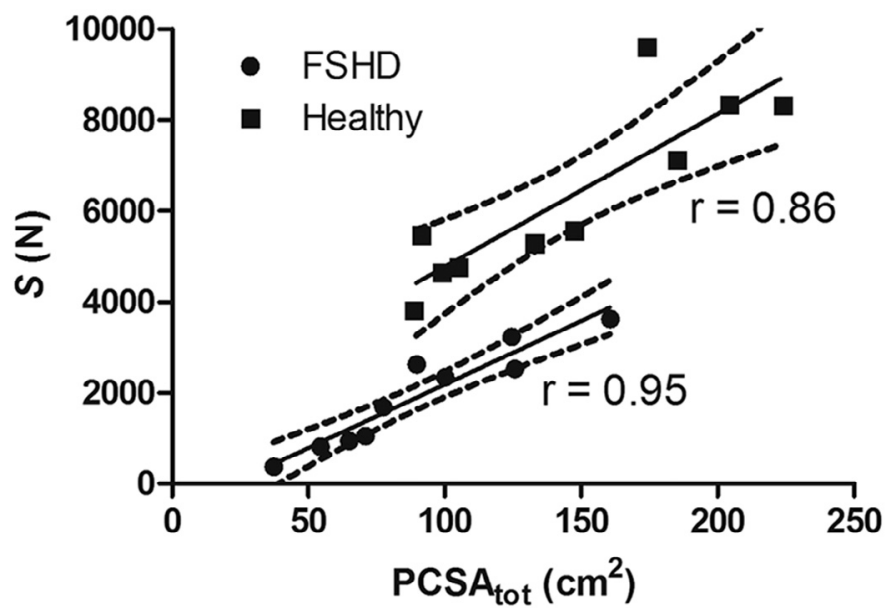

Fig. 3. Relationship between quadriceps muscle strength $(S)$ and total physiological cross-sectional area $\left(\mathrm{PCSA}_{\text {tot }}\right)$ in patients with facioscapulohumeral dystrophy (FSHD) and healthy controls. 


\section{Specific Strength}

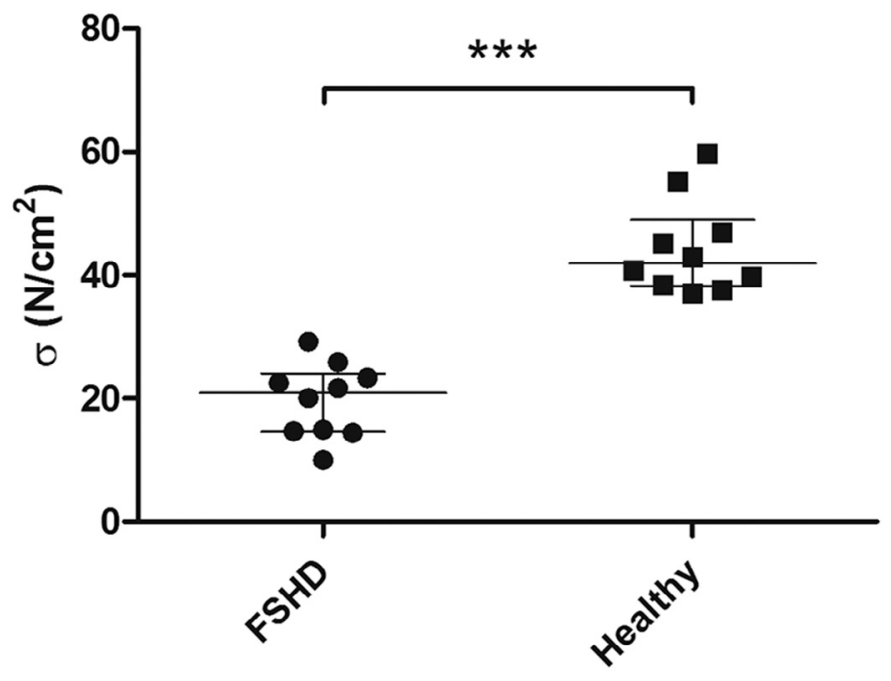

Fig. 4. Reduced specific strength $(\sigma)$ of quadriceps muscles in patients with facioscapulohumeral dystrophy (FSHD) and healthy controls. Auxiliary lines indicate median (1st quartile-3rd quartile). Asterisks $(* * *)$ indicate $\mathrm{p}<0.001$.

specific muscle strength is reduced in FSHD patients. This reduced specific muscle strength is not unique to FSHD, as similar results have been reported for Duchenne $[16,17]$ and Becker muscular dystrophy patients [18]. The exact reason why specific muscle strength is reduced in FSHD is unknown. Potential explanations may be related to changes at the level of muscle fibers, energy metabolism, or in cytoarchitecture, resulting in biomechanical changes that alter force propagation.

First, reduced specific strength of the quadriceps may reflect reduced strength generation of individual muscle fibers. A pilot study by Lassche et al. [8] found a 30\% strength reduction in demembranated FSHD type II fibers obtained from quadriceps muscle biopsies from FSHD patients, compared with healthy controls. Second, the integrity of the sarcolemma can be compromised in FSHD muscle fibers, which may hamper lateral transmission of force generated by muscle fibers [19]. Third, mitochondria in FSHD quadriceps muscle tissue might be dysfunctional [20]. Furthermore, in vivo measurement with MR spectroscopy showed that phosphocreatine to ATP ratio is reduced in moderately fat infiltrated FSHD muscles, indicating that energy metabolism is indeed altered $[3,21]$. Both the ATP synthesis [20] and the ratio of phosphocreatine to ATP [3] correlate positively with the force produced by the quadriceps muscles. Fourth, fatty infiltration may hamper the transfer of force to the tendon due to fatty interposition, resulting in reduced muscle strength. Advanced imaging techniques, such as ultrasound strain measurement [22-24] and diffusion-tensor imaging [25] can demonstrate muscle contraction patterns and force propagation and may characterize possible alterations in muscle fibers in FSHD. Besides fatty infiltration, FSHD muscles may suffer from increased interstitial fibrosis which would reduce the fractional muscular area. As our MRI protocol is not designed to detect fibrosis it was not taken into account in our study. As a consequence, the contractile muscle volume might have been overestimated, resulting in underestimation of the specific muscle strength in FSHD in our study. However, to explain the $\pm 50 \%$ reduction of specific muscle strength in our group of FSHD patients compared to healthy controls, the remaining contractile muscle volume should consist for $50 \%$ of fibrosis. In humans fractional fibrotic areas are rarely quantified. For the heart it has been reported that myocardial fibrosis may be present up to $15 \%$ in coronary disease [26]. In an FSHD mouse model extreme high induction of DUX4 resulted in approximately 15\% fibrosis in the gastrocnemius [27]. As our patients only showed a mild to moderate fat infiltration it is unlikely that fibrosis has contributed significantly to the lower specific muscle strength observed in our study.

Quadriceps fat fraction in this study varied from normal $(<10 \%)$ to moderately affected $(46 \%)$. This was reflected by the clinical severity scores. Three patients had no lower extremity involvement and one patient was unable to walk unaided. Thus, FSHD patients in our study show a large variability as is also seen in the FSHD population in general. Due to the retrospective nature of this study, the FSHD patients and healthy controls were recruited from two different cohorts in which a different force measurement device and MR protocol was used. Our post-hoc experiment comparing the two measurement devices in a separate group of healthy volunteers showed no systemic difference between the two devices. The MR protocol in healthy volunteers did not allow quantitative assessment of fat infiltration. Therefore, the fat fractions for healthy controls used in this study were obtained from a separate group of healthy volunteers measured with the same 2pt Dixon protocol as the FSHD patients. For this reason, we conclude that the reduction of specific strength is not due to the different measurement set-up and MR protocol used in the FSHD patients and healthy controls. Since FSHD patients may have been fatigued or felt pain [28] more than healthy volunteers, this might have lowered their MVIC measurement. However, Bacchasson et al. [7] found similar central and peripheral quadriceps fatigability in FSHD patients vs. controls, suggesting that fatigue or pain did not cause the changes in specific strength. In our musculoskeletal model the pennation angle and optimal muscle fiber length were assumed to be similar in FSHD patients and healthy controls, although these may have been altered by disease processes in FSHD. However, measuring optimal fiber length and pennation angle in vivo is not feasible in a clinical setting, if not even impossible with the current techniques. Finally, the rectus femoris was included in the total quadriceps muscle PCSA, even though it has a minor contribution to force generation in the sitting position analyzed. However, in our FSHD cohort the rectus femoris is the most fat infiltrated quadriceps muscle. Thus, if the rectus femoris contribution is not taken into account the difference in specific strength between the two groups would become even larger.

This study may serve as a reference for future studies employing computer models of the musculoskeletal systems to study FSHD, as it provides values of specific muscle strength of an FSHD group in comparison to a group of healthy controls. 
Incorporating FSHD-specific model parameters, such as specific muscle strength, is important as the recruitment of muscles is dependent on the capacity of each muscle to generate force. To the present, it remains unclear, for instance, whether FSHD would also alter the recruitment of muscles during daily activities, which warrants further investigation.

\section{Conclusions}

Specific muscle strength of the quadriceps muscle is reduced in a group of FSHD patients compared to healthy controls, suggesting an intrinsic impairment in the force-generating capacity, energy metabolism and/or force propagation in FSHD muscles. Total contractile PCSA is also reduced in FSHD muscles compared to healthy controls, indicating that fatty infiltration and atrophy are co-factors associated with muscle weakness in FSHD. This finding is important for two reasons. First, future studies that apply musculoskeletal models in FSHD patients should consider reduced specific muscle strength. Second, it tells us that interventions targeting muscle weakness in FSHD should focus on treating or preventing not only fatty infiltration and changes in contractile muscle volume, but also aim to restore specific muscle strength.

\section{Funding}

This work was supported by the European Research Council under the European Union's Seventh Framework Programme (FP/2007-2013), grant numbers 247860 and 323091, awarded to N. Verdonschot. The FSHD-FOCUS study was funded by the Prinses Beatrix Spierfonds and Spieren voor Spieren. Furthermore, this work is supported by the European Community's Seventh Framework Programme (FP7/2007-2013), grant number 305697, and by the FSHD stichting Nederland.

\section{Acknowledgments}

We are thankful to Mike Kattenbelt (Sint Maartensliniek Sport Medisch Centrum, Nijmegen, The Netherlands) for his kind assistance during the experimental measurements with the Biodex device. Furthermore, we would like to thank Niala den Braber, Dieuwke van Dartel and Job Stoks for their help in the processing and analysis of the data.

\section{References}

[1] Mul K, Lassche S, Voermans NC, Padberg GW, Horlings CG, van Engelen BG. What's in a name? The clinical features of facioscapulohumeral muscular dystrophy. Pract Neurol 2016;16:201-7.

[2] Dubowitz V, Sewry C. Muscle biopsy: a practical approach. 2007.

[3] Janssen BH, Voet NBM, Nabuurs CI, Kan HE, de Rooy JWJ, Geurts AC, et al. Distinct disease phases in muscles of facioscapulohumeral dystrophy patients identified by MR detected fat infiltration. PLoS ONE 2014;9:e85416.

[4] Leung DG, Carrino JA, Wagner KR, Jacobs MA. Whole-body magnetic resonance imaging evaluation of facioscapulohumeral muscular dystrophy. Muscle Nerve 2015;52:512-20.

[5] Kan HE, Scheenen TWJ, Wohlgemuth M, Klomp DWJ, van LoosbroekWagenmans I, Padberg GW, et al. Quantitative MR imaging of individual muscle involvement in facioscapulohumeral muscular dystrophy. Neuromuscul Disord 2009;19:357-62.

[6] Spector SA, Gardiner PF, Zernicke RF, Roy RR, Edgerton VR. Muscle architecture and force-velocity characteristics of cat soleus and medial gastrocnemius: implications for motor control. J Neurophysiol 1980;44: 951-60.

[7] Bachasson D, Temesi J, Bankole C, Lagrange E, Boutte C, Millet GY, et al. Assessement of quadriceps strength, endurance and fatigue in FSHD and CMT: benefits and limits of femoral nerve magnetic stimulation. Clin Neurophysiol 2014;125:396-405.

[8] Lassche S, Stienen GJM, Irving TC, van der Maarel SM, Voermans NC, Padberg GW, et al. Sarcomeric dysfunction contributes to muscle weakness in facioscapulohumeral muscular dystrophy. Neurology 2013;80:733-7.

[9] Ricci E, Galluzzi G, Deidda G, Cacurri S, Colantoni L, Merico B, et al. Progress in the molecular diagnosis of facioscapulohumeral muscular dystrophy and correlation between the number of $\mathrm{KpnI}$ repeats at the 4q35 locus and clinical phenotype. Ann Neurol 1999;45:751-7.

[10] Lund H, Christensen L, Savnik A, Boesen J, Danneskiold-Samsøe B, Bliddal H. Volume estimation of extensor muscles of the lower leg based on MR imaging. Eur Radiol 2002;12:2982-7.

[11] Damsgaard M, Rasmussen J, Christensen ST, Surma E, de Zee M. Analysis of musculoskeletal systems in the AnyBody Modeling System. Simul Model Pract Theory 2006;14:1100-11.

[12] Ward SR, Eng CM, Smallwood LH, Lieber RL. Are current measurements of lower extremity muscle architecture accurate? Clin Orthop Relat Res 2009;467:1074-82.

[13] Sherman MA, Seth A, Delp SL. What is a moment arm? Calculating muscle effectiveness in biomechanical models using generalized coordinates. Vol 7B 9th Int Conf Multibody Syst Nonlinear Dyn Control, vol. 2013, ASME; 2013, p. V07BT10A052.

[14] Sacks RD, Roy RR. Architecture of the hind limb muscles of cats: functional significance. J Morphol 1982;173:185-95.

[15] O’Brien TD, Reeves ND, Baltzopoulos V, Jones DA, Maganaris CN. In vivo measurements of muscle specific tension in adults and children. Exp Physiol 2010;95:202-10.

[16] Akima H, Lott D, Senesac C, Deol J, Germain S, Arpan I, et al. Relationships of thigh muscle contractile and non-contractile tissue with function, strength, and age in boys with Duchenne muscular dystrophy. Neuromuscul Disord 2012;22:16-25.

[17] Wokke BH, van den Bergen JC, Versluis MJ, Niks EH, Milles J, Webb AG, et al. Quantitative MRI and strength measurements in the assessment of muscle quality in Duchenne muscular dystrophy. Neuromuscul Disord 2014;24:409-16.

[18] Løkken N, Hedermann G, Thomsen C, Vissing J. Contractile properties are disrupted in Becker muscular dystrophy, but not in limb girdle type 2I. Ann Neurol 2016;80:466-71.

[19] Reed P, Porter NC, Strong J, Pumplin DW, Corse AM, Luther PW, et al. Sarcolemmal reorganization in facioscapulohumeral muscular dystrophy. Ann Neurol 2006;59:289-97.

[20] Turki A, Hayot M, Carnac G, Pillard F, Passerieux E, Bommart S, et al. Functional muscle impairment in facioscapulohumeral muscular dystrophy is correlated with oxidative stress and mitochondrial dysfunction. Free Radic Biol Med 2012;53:1068-79.

[21] Kan HE, Klomp DWJ, Wohlgemuth M, van Loosbroek-Wagemans I, van Engelen BGM, Padberg GW, et al. Only fat infiltrated muscles in resting lower leg of FSHD patients show disturbed energy metabolism. NMR Biomed 2010;23:563-8.

[22] Gijsbertse K, Sprengers AMJ, Nillesen MM, Hansen HHG, Lopata RGP, Verdonschot N, et al. Three-dimensional ultrasound strain imaging of skeletal muscles. Phys Med Biol 2017;62:596-611.

[23] Lopata RGP, van Dijk JP, Pillen S, Nillesen MM, Maas H, Thijssen JM, et al. Dynamic imaging of skeletal muscle contraction in three orthogonal directions. J Appl Physiol 2010;109:906-15.

[24] Gijsbertse K, Goselink R, Lassche S, Nillesen M, Sprengers A, Verdonschot $\mathrm{N}$, et al. Ultrasound imaging of muscle contraction of the tibialis anterior in patients with facioscapulohumeral dystrophy. Ultrasound Med Biol $2017 ; 1-9$ 
[25] Oudeman J, Mazzoli V, Marra MA, Nicolay K, Maas M, Verdonschot N, et al. A novel diffusion-tensor MRI approach for skeletal muscle fascicle length measurements. Physiol Rep 2016;4:e13012.

[26] de Meester de Ravenstein C, Bouzin C, Lazam S, Boulif J, Amzulescu M, Melchior J, et al. Histological Validation of measurement of diffuse interstitial myocardial fibrosis by myocardial extravascular volume fraction from Modified Look-Locker imaging (MOLLI) T1 mapping at 3 T. J Cardiovasc Magn Reson 2015;17:48.
[27] Bosnakovski D, Chan SSK, Recht OO, Hartweck LM, Gustafson CJ, Athman LL, et al. Muscle pathology from stochastic low level DUX4 expression in an FSHD mouse model. Nat Commun $2017 ; 8: 550$

[28] Kalkman JS, Schillings ML, van der Werf SP, Padberg GW, Zwarts MJ, van Engelen BGM, et al. Experienced fatigue in facioscapulohumeral dystrophy, myotonic dystrophy, and HMSN-I. J Neurol Neurosurg Psychiatry 2005;76:1406-9. 